\title{
Role of verbal encoding in short- and long-term odor recognition
}

\author{
C. JEHL, J.-P. ROYET, and A. HOLLEY \\ Université Claude-Bermard, Villeurbanne, France
}

\begin{abstract}
The role of verbal encoding in odor recognition memory was investigated using odors of low familiarity to subjects before the experiment began. The experimental procedure included two phasesodor learning (first phase) and odor memory testing (second phase)—separated by a delay of 7 days. Five experimental conditions were established: three conditions of odor learning with names (labeling conditions), one condition of odor learning without names (sensory familiarization), and one condition of no learning prior to testing (control conditions). The labeling conditions differed from each other regarding label characteristics. The names were those of odor sources (veridical names), those personally generated by subjects (generated names), or those derived from the chemical names of the odorants (chemical names). Subjects were required to learn 20 fixed associations between odors (targets or distractors) and 20 names during two daily sessions. The learning sessions included two identification tests and ended by a verbal memory test in which subjects recalled odor names. The odor memory test was split into two parts separated by a retention interval of either 20 min (short-term memory) or $24 \mathrm{~h}$ (long-term memory). Data showed that olfactory recognition memory was enhanced in subjects who associated veridical or generated names to odors during the learning session. Chemical names were not appropriate to facilitate odor recognition. Similarly, the level of odor identification was higher for veridical and generated names than for chemical names, though the level of verbal memory for chemical names was substantial. Recognition response latencies were systematically longer for a target odor implying a positive response than for a distractor odor implying a negative response. Together, these data suggest that odor recognition and identification are sensitive to the semantic content of labels associated with odors. Odor memory was adversively influenced by time, but this influence was less pronounced when the names were endowed with a rich semantic content.
\end{abstract}

In the verbal domain, current theories assume that the durability of the memory trace is dependent on the level to which a verbal item is processed at the time of encoding (Craik \& Lockhart, 1972). Accordingly, a deeper level of encoding is generally linked with semantic processing of verbal information, whereas a shallower encoding level is related to perceptual processing of that information. Hence, it is a common notion that the semantic processing of verbal items is associated with higher memory performance.

In the olfactory domain, until recently, there was a controversy over whether the encoding of odors could be efficiently mediated by semantic cues (Engen, 1987; Richardson \& Zucco, 1989; Schab, 1991). It was thought that odors were encoded as unitary perceptual events (Engen, Kuisma, \& Eimas, 1973; Engen \& Ross, 1973; Lawless, 1978). This controversy stemmed from the following observations. First, the link between odors and their names is generally weak compared with the strong one existing between pictures and names (Engen, 1987; Shepard, 1967). Hence, the association between new names and familiar odors or between common names and unfamiliar odors

Correspondence should be addressed to J.-P, Royet, Université Claude-Bernard, Laboratoire de Physiologie Neurosensorielle, 43, Bd. du 11 novembre 1918, 69622 Villeurbanne Cedex, France (e-mail: royet@neurosens.univ-lyon1.fr). proved to be a difficult task (Cain, 1980; Davis, 1977). This may explain in part why odors are described using an idiosyncratic language rather than by their appropriate names (Engen, 1982, 1987). Second, the level of odor identification in absence of training, even for relatively familiar odors, is relatively low (Murphy, 1985). One may assume that odors are not spontaneously perceived as individual entities, in the absence of deliberate training, and remain associated with the context of exposure. The identification of odor becomes challenging if several experiences of the same odor in different contexts have to be designated by a single descriptor. As an example, "clove" is the odor of a spice or sometimes an odor encountered at the dentist's.

Among the previous works to be indicative of the possibility for odor encoding to be mediated through verbal information is that by Rabin and Cain (1984). These authors uncovered a relationship between identifiability in terms of encoding consistency (i.e., to associate a fixed verbal label to the same odor) and olfactory recognition memory. In a yes-no recognition paradigm study, it was shown that verbal mediation to target odors (the to-beremembered odors), by way of associating names to them during the retention interval, was favorable to short-term (26-sec) retention of these odors (Walk \& Johns, 1984). In contrast, the same kind of mediation to distractor odors impaired retention of the targets. Walk and Johns inter- 
preted their findings as a consequence of the negative interference of verbal mediation to distractors upon the memorization of targets. Thus, this kind of verbal interference suggests that the perceptual code for odors can be augmented by a verbal code.

Another study by Lyman and McDaniel (1986) showed that different elaboration activities made on targets, during the inspection phase, would produce differential effects on the long-term (1-week) retention of these odors. The authors' finding showed that subjects instructed to associate a label plus definition to the targets (verbal elaboration) or those referring a life episode to them exhibited higher memory performance than did subjects instructed to form a mental image of the target source (visual image elaboration) and those making no elaboration at all. This result supports the notion that long-term memory for odors is enhanced by verbal encoding.

The dual coding theory by Paivio (1986) assumes that an item can be encoded in a verbal representational system, in a nonverbal representational system, or in both. Thus, this theory supposes that items being twice coded have a greater chance to be retrieved from memory than do those once coded. Lyman and McDaniel (1986) found that recognition memory was better for odors encoded by two additional codes - a verbal one and a visual oneas compared with odors encoded by only one code - verbal or visual-added to the olfactory code. The authors inferred from their results that additional codes produced more retrieval pathways for odor memory, and, hence, odor memorization was enhanced.

The general objective of this investigation was to further document the role of verbal encoding in odor memory. We wanted to investigate the effect of learning associations between odors and labels on odor recognition memory. Because associations could not be learned without repeated exposure to odors, it was necessary to dissociate odor labeling from pure familiarization to odors. A group of subjects was submitted to a pure familiarization procedure with odors that were not given labels. In addition, we made the assumption that the mnemonic effectiveness of verbal labels, acting as retrieval cues for odors, would depend on the degree of their semantic precision. For instance, veridical labels and labels generated by the subjects themselves were expected to be highly denotative of specific odor sources, when compared with chemical names that are only slightly meaningful to the subjects. Finally, the subjects were also tested for their ability to associate specific names to odors - that is, to identify odors.

Several experimental conditions were assessed. First, odors of relatively low familiarity were preferably used as stimuli because it was supposed that these odors had no precise mnemonic representations. It is worth noting that highly familiar odors (i.e., food and household products) served as stimuli in previous studies on the role of verbal coding in olfactory memory (Lyman \& McDaniel, 1986, 1990; Walk \& Johns, 1984). These kinds of odors could have received some verbal labeling prior to the experiment, even though this labeling was not precise, and resulted in denominations denoted as "near misses" or "far misses" (Cain, 1979; Rabin \& Cain, 1984). As a consequence, it seemed difficult to measure with high precision the influence of experimental conditions of labeling on odor retention. In contrast, when initially unfamiliar odors are used as stimuli, the learning of their labels could equate this source of variability across subjects. The learning of associations between odors and names was intensive in order to ensure that all odors being sampled were linked to a label. Second, in previous studies, the subjects were generally instructed to attach verbal labels to the targets but not to the distractors. This raises the question of whether targets were discriminated from distractors on the basis of a distinction between a newly presented odor (distractor) and an odor already experienced (target) or in terms of verbal labels. To clear up this ambiguity, we preferred having subjects trained to learn fixed associations between odors and names for both targets and distractors.

\section{METHOD}

\section{Subjects}

One hundred subjects ( 50 women, 50 men; $18-32$ years of age, mean $=25.3$ years) volunteered to serve in this experiment. The majority were thesis students, and others were college graduate students enrolled in neuroscience or biology courses. Prior to this study, none had participated in an olfactory experiment. All were nonsmokers. None presented either hyposmia or anosmia to tested odors. During their menstrual cycle, only 2 women were found to be in the ovulatory period, which has been described as the period in which women proved to be more sensitive to odors (Doty, Snyder, Huggins, \& Lowry, 1981; Schneider, 1974). It is possible that several cases of ovulation were ignored. Additionally, except the odors of soap or deodorant that were considered not to have a strong odor, no perfumes were permitted throughout testing. The subjects were paid for their participation.

\section{Stimuli}

The stimulus sample included 20 odorants. It spanned a wide range of qualities representative of a large variety of olfactory notes (see Table 1): floral, fruity, citrusy, woody, musky, spicy, camphoraceous, aromatic, anisic, and green. All odorants were pure chemicals. They were chosen so as to represent low initial familiarity, because familiarity has been shown to interfere with olfactory recognition memory (Jehl, Royet, \& Holley, 1995). They were also selected as being representative of near-neutral pleasantness. Some of them were furnished by Givaudan (Grasse, France), and others were furnished by Aldrich chemistry products (Strasbourg, France).

The odors were contained in 120 -ml yellow glass jars with screw lids. These jars were opaque in order to ensure that the subjects had no possibility of relying upon visual cues to identify odors. Each jar contained $10 \mathrm{ml}$ of a solution. Mineral oil served as the solvent. Throughout testing, all odors were of moderate intensity to prevent the subjects from sensory adaptation. To obtain homogeneous subjective intensities across odor presentations, various concentrations were needed. Hence, the odors of lower intensity, such as coumarin, lilial, and ambrettolide, were diluted 10 times; other ones, such as trimethylcyclohexanone and phenylethyl alcohol, were diluted 100 times. Hydratropic aldehyde was diluted 1,000 times, because this chemical released a very strong odor. These concentrations were established using pilot subjects. The levels of odor concentration were determined by comparing each odor with a standard odorant, ( $\beta$-phenylethanol, chosen arbitrarily at a given level of concentration. The respective grades of dilution of all odors are listed in 
Table 1

List of the Chemical Substances Used in This Study by Chemical Name and Common Name With Their Corresponding Grades of Dilution

\begin{tabular}{llrr}
\hline \multicolumn{1}{c}{$\begin{array}{c}\text { Chemical } \\
\text { Name }\end{array}$} & \multicolumn{1}{c}{$\begin{array}{c}\text { Veridical } \\
\text { Name }\end{array}$} & Set & $\begin{array}{r}\text { Dilution } \\
\text { Factor }\end{array}$ \\
\hline Anis aldehyde & $\begin{array}{l}\text { Target } \\
\text { anisic-spicy }\end{array}$ & 1 & \\
Cedryl acetate & cedar wood & 1 & 500 \\
Coumarin & nuts & 3 & 10 \\
Para cymene & light-citrusy & 4 & 100 \\
Beta ionone & violet & 4 & 10 \\
Linalool & coriander wood & 1 & 100 \\
Methyl cinnamate & cinnamate & 1 & 50 \\
Safrole & fennel & 2 & 100 \\
Sandela & sandalwood & 3 & 10 \\
3.3.5. Trimethylcyclohexanol & camphoraceous & 4 & 100 \\
& Distractor & & \\
Ambrettolide & musky-blackberry & 2 & 10 \\
Base 3 & green pepper & 3 & 1,000 \\
Cumaldehyde & cumin & 3 & 1,000 \\
Diamyl ketone & pineapple & 2 & 100 \\
Beta hexenol & green grass & 4 & 1,000 \\
Hydratopic aldehyde & honey flower & 4 & 1,000 \\
d-Limonene & orange & 2 & 50 \\
Lilial & lilac & 2 & 10 \\
$\beta$-Phenylethyl alcohol & rose & 1 & 100 \\
Prunolide & coconut & 3 & 50 \\
\hline
\end{tabular}

Table 1. Odorants were kept in a refrigerator when not used; they were taken out of the refrigerator before the experiment began and left to reach room temperature.

\section{Experimental Design}

The subjects were run in 10 groups that differed according to the labeling and memory-testing conditions (short and long retention intervals) to which they were assigned. In order to examine the influence of the quality of odor names on the subjects' abilities to consistently label an odor using the same name, three pairs of groups differed from each other by the quality (the degree of meaningfulness) of the names attached to odors: chemical-name groups, generatedname groups, and veridical-name groups. Two pairs of groups served as controls: no-learning groups and no-label groups.

Chemical-name groups. The subjects were trained to learn 20 single fixed associations between the test odors and names that were simplified forms of their chemical names. These names were less meaningful to the subjects than were veridical and personally generated ones.

Generated-name groups. The subjects were trained to learn 20 single fixed associations between test odors and names that were generated by the subjects. The subjects were allowed to spend as much time as needed to utter a name for an odor. The experimenter then wrote the name announced by the subjects on a 5 -in. card.

Veridical-name groups. The subjects were trained to learn 20 single fixed associations between test odors and veridical names. For example, the name "rose" was uttered for the compound $\beta$ phenylethanol during the learning sessions.

No-learning groups. The subjects in these control groups participated only in the second part of the experiment (i.e., they were tested for memory performances without any previous learning or familiarization). These groups were employed to assess any possible disadvantage of the no-training condition over the training condition.

No-label groups. The subjects in these control groups were familiarized with test odors throughout learning sessions. They were exposed to odorants as many times as were the labeling groups, but no names were associated to odors. They were instructed to smell odors in silence.

\section{Procedure}

The whole experiment included three sessions (see Figure 1). In the first two sessions, the subjects were required to perform a task that consisted of learning fixed associations between 20 odors of the test sample and 20 names. In the third session, the influence of verbal labeling on subjects' recognition memory performance was assessed. Two conditions were used for testing recognition memory: short and long retention intervals. Furthermore, the odor identification tests (odor naming) and the verbal memory test (odor-name recall) were performed during the three sessions. Sessions 1,2 , and 3 lasted $2 \mathrm{~h}$ and $30 \mathrm{~min}, 1 \mathrm{~h}$ and $45 \mathrm{~min}$, and $30 \mathrm{~min}$, respectively.

Learning sessions. A trial consisted of matching an odor of a set to a name provided by the experimenter. The experimenter first presented the subjects with an odor and then uttered a name for it while presenting a card on which the uttered name was written in large blue letters. Afterwards, the subjects were allowed to write that name on a piece of white paper in order to aid memory processes and, thus, to overcome difficulties in retrieval of that name. On subsequent trials of an odor, the experimenter only uttered the name announced on the first trial. To strengthen learning of verbal associations to odors, the subjects had their learning task split between two different and successive daily sessions (Figure 2).

First session: The first session consisted of a total of 80 trials to match the 20 odors to 20 names. All subjects performed four blocks of odor-name matching. Each block consisted of four successive presentations of the same set(s) of 5 odors (four sets $S 1$ in Block 1 , four sets $S 2$ in Block 2, etc.). The presentation of a set comprised five different trials. The distribution of odors among the four sets is indicated in Table 1. There was no rule for including any odor in a set, only a random choice. The order of odors was different among the five sets of the same block. The interstimulus interval between trials and sets was $45 \mathrm{sec}$. During this interval, the subjects were instructed to memorize the association between a name and an odor while being silent. Five-minute respites separated successive blocks in order to counteract fatigue and sensory adaptation across odors.

Second session: The second daily session differed from the first one in two respects. First, the to-be-learned odors were distributed into only one set of 20 odors for a total of 40 trials (i.e., 2 trials per odor). Second, there were only two presentations of this set. Thus, the subjects strengthened their learning of the 20 fixed associations between odors and their respective names.

Identification tests. There were three identification tests

First identification test $\left(I T_{1}\right)$ : On the fifth presentation of a set for each block of Session 1 (Figure 2), the experimenter successively delivered the 5 odors and asked the subjects if they could retrieve the names that had previously been attached to these odors. At the beginning of the first session, the subjects had been instructed that they would be tested for their ability to retrieve the correct name of the odors. They were asked to respond as quickly as possible; the experimenter recorded their responses. They were told their score of correct responses and were given corrective feedback regarding the name they had provided for each odor.

Second identification test $\left(I T_{2}\right)$ : On the third presentation of the set of 20 odors in the second session, all subjects were instructed to identify odors by their uttered names with no help from cards. The experimenter recorded their responses for each of the 20 trials. Again, the subjects were given feedback with the correct names and were told their scores of correct responses.

Third identification test $\left(I T_{3}\right)$ : The same procedure as that for the $\mathrm{IT}_{2}$ part of second session was replicated in the third session 1 week later. 

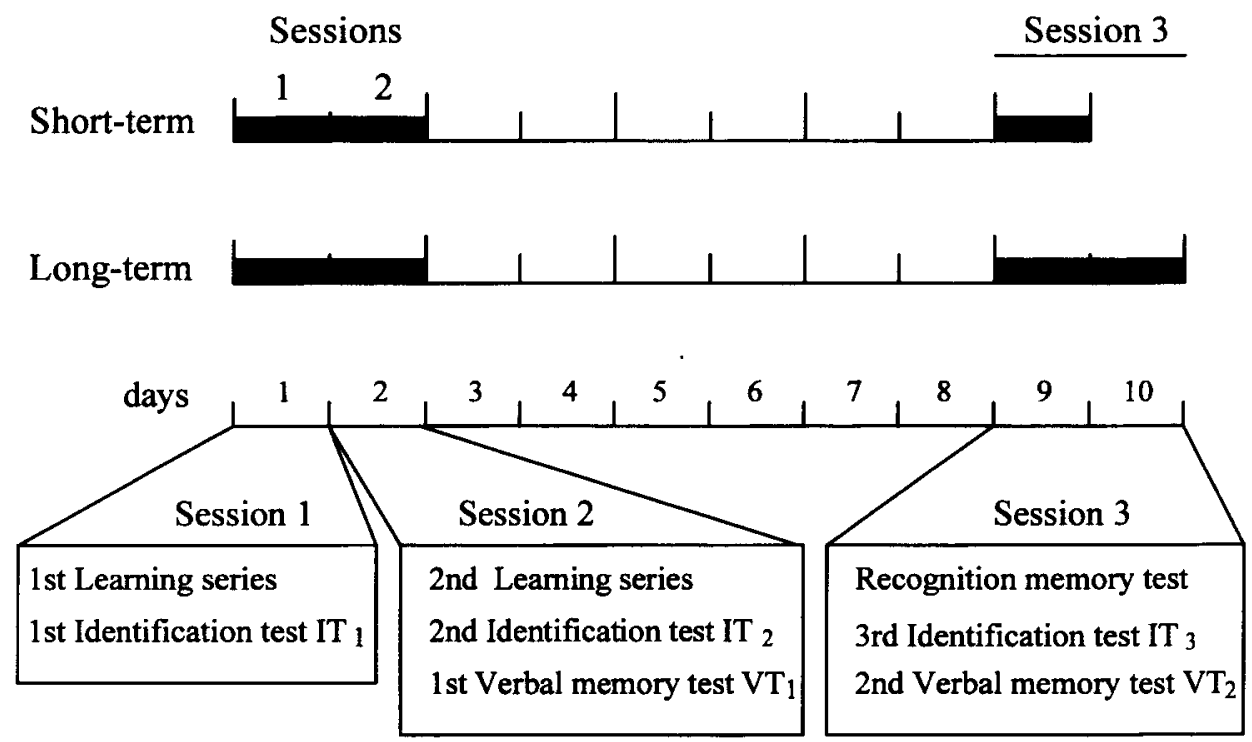

Figure 1. Diagram of the chronology of the experimental procedure. The first part included learning of verbal associations to odors during Sessions 1 and 2 . At the end of Session 1 , the subjects performed the first identification test $\left(I T_{1}\right)$; at the end of Session 2, they performed the second identification test $\left(\mathrm{IT}_{2}\right)$ and the first verbal memory test $\left(\mathrm{VT}_{1}\right)$. The second part (Session 3 ), which began 1 week after Learning Session 2, included the odor recognition memory test, the third identification test (IT ( $_{3}$, and the second verbal memory test $\left(\mathrm{VT}_{2}\right)$. Recognition memory was studied in two conditions: shortterm (20-min) and long-term (24-h) memory.

Recognition memory test. Two yes-no recognition tests took place 1 week following the learning phases of the first two sessions: one short-term and one long-term memory test (Figure 1). This delay of 7 days was aimed at preventing the subjects from confusion in determining whether the target odors were delivered during the learning sessions or the recognition memory test. The recognition memory test was built up in two parts separated from each other by a delay that served as a retention period. For the five short-term memory groups, it lasted $20 \mathrm{~min}$; for the long-term memory groups, it lasted $24 \mathrm{~h}$. The short-term memory test was performed on Day 9; the long-term memory test was performed on Days 9 and 10 . Throughout the first part of the memory test, all subjects were first presented with 10 target odors chosen among the 20 learned odors. The test odors were presented in the same order across all subjects. On the second part of the memory test (i.e., $20 \mathrm{~min}$ or $24 \mathrm{~h}$ later), the subjects were presented with 20 odors, including 10 targets and 10 distractors (i.e., odors not previously delivered during the first part of the test). The distractors were those odors remaining from the learned sample after selection of the targets. Targets and distractors presented, therefore, the same degree of familiarity to the subjects. Targets were randomly interspersed among the distractors. The 20 odors followed each other in a regular order every $45 \mathrm{sec}$ across subjects. After delivery of an odor, the subjects responded "yes" or "no" according to whether or not the odor had been delivered during the first part of the test. They were informed that response latency would be recorded. This was done with the aid of a chronometer. Inspection of the jars containing either a target or a distractor odor lasted $5 \mathrm{sec}$.

Verbal memory test. There were two verbal memory tests.

First verbal memory test $\left(V T_{1}\right)$ : When the second identification test $\left(\mathrm{IT}_{2}\right)$ of Session 2 was over, the subjects were requested to write, on a piece of white paper, all the names assigned to the test odors that they were able to retrieve from memory. To perform this task, they spent as much time as desired.

Second verbal memory test $\left(V T_{2}\right)$ : At the end of Session 3, a test identical to $\mathrm{VT}_{1}$ was performed.

\section{RESULTS}

\section{Quantitative and Statistical Analyses}

Recognition memory performance was assessed using parameters issued from the signal detection theory (Banks, 1970; Lockhart \& Murdock, 1970). For identical-odor pairs, a hit was scored if the two odors were recognized as "identical" by the subject, and a miss was scored if these two odors were incorrectly judged as "different." For dissimilar-odor pairs, a correct rejection was scored if the two odors were recognized as "different" and a false alarm was scored if these two odors were judged as "identical." From hit and false-alarm scores, four parameters were deduced-hit rate, false-alarm rate, discrimination measure, and response bias-as described by Snodgrass and Corwin (1988). To analyze recognition scores, general methods of one-, two-, and three-way analyses of variance (ANOVAs), with or without repeated measurements, were used (Winer, 1962). Differences between pairs or groups of means were assessed by multiple orthogonal contrasts. The normality of the samples and the homogeneity of their variance were controlled with the Lilliefors test (Conover, 1971) and the Hartley test (Winer, 1962), respectively.

\section{Recognition Performance as a Function of Labeling}

Figure 3 represents the arithmetic mean of hit scores, false-alarm scores, discrimination measures, and responsebias measures as a function of the labeling factor (nolearning, no-label, chemical-name, generated-name, and 


\section{$\begin{array}{llll}\text { Block } 1 & \text { Block } 2 & \text { Block } 3 & \text { Block } 4\end{array}$}
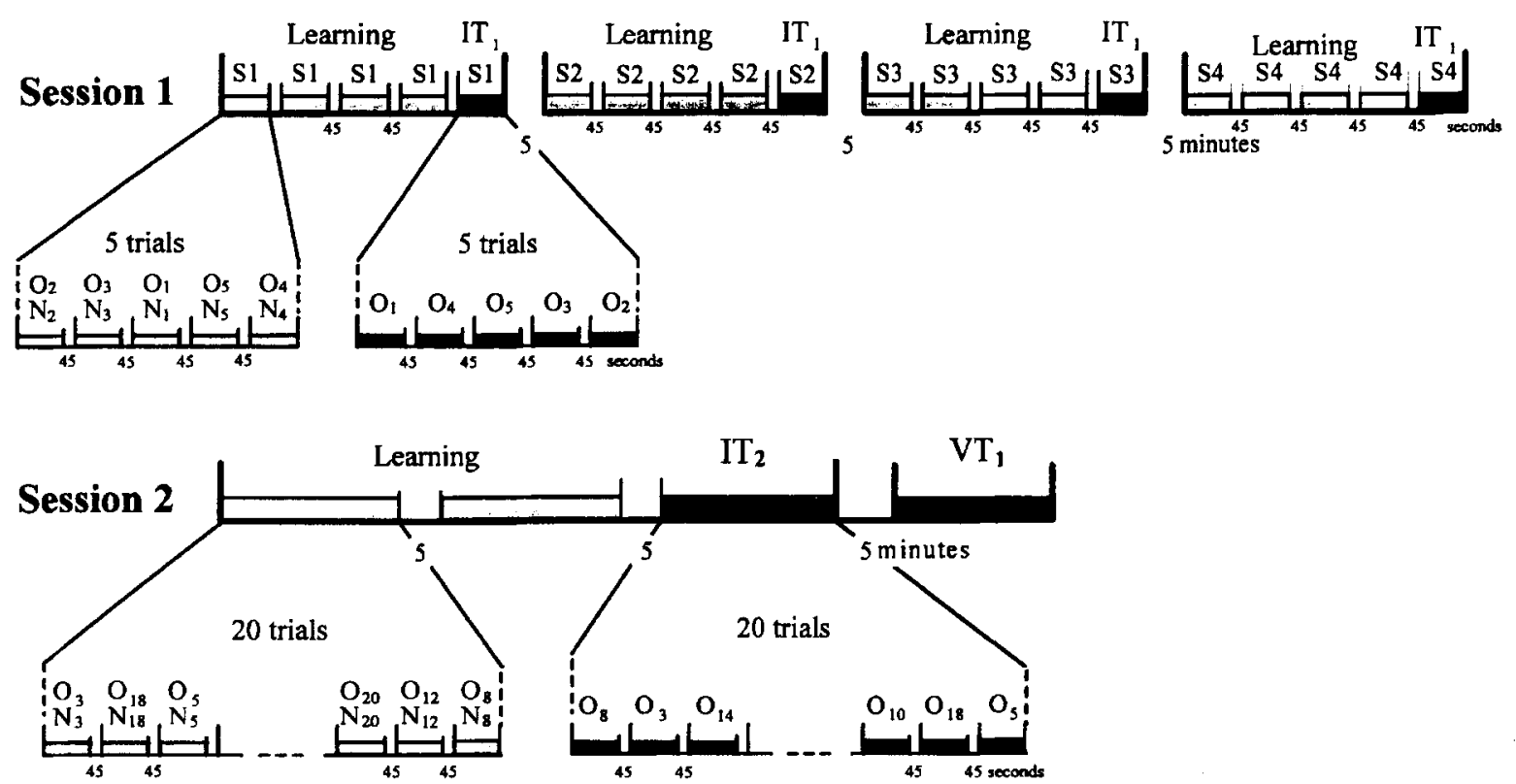

Figure 2. Detailed representation of the first part of the experimental procedure. In Session 1, the subjects were presented

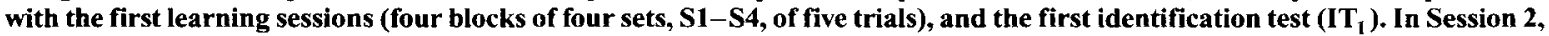
the subjects were presented with the second learning sessions (two times a set of 20 odors), the second identification test (IT ${ }_{2}$ ), and the first verbal memory test $\left(V T_{1}\right)$. O, odor; $N$, name. See the text for detailed explanations.

veridical-name conditions) and as a function of the type of memory tested (short-term and long-term).

The two-way ANOVA revealed significant differences of hit scores for the labeling condition $[F(4,90)=10.72$, $p<.0005]$ and for the memory condition $[F(1,90)=$ $16.07, p<.0005]$, but no significant interaction between these two factors $[F(4,90)=2.12$, n.s.]. As shown by multiple orthogonal contrasts, these significant differences were due to hit scores of no-label and chemicalname conditions being lower than those of the other labeling conditions in the case of long-term memory ( $p<$ .0005 , for all comparisons by pairs of means). A lower score was also observed for the chemical-name condition relative to the veridical-name condition in short-term memory $[F(1,90)=10.73, p<.005]$.

For false-alarm scores, the two-way ANOVA showed a highly significant effect of the labeling condition $[F(4,90)=4.91, p<.005]$, no effect of the memory condition $[F(1,90)=3.07$, n.s. $]$, and no interaction between both factors $[F(4,90)=1.49$, n.s. $]$. As evidenced by multiple orthogonal comparisons between means, the significant effect of the labeling factor was due to a number of false alarms being significantly higher in long-term memory for the no-label condition than for the no-learning condition $[F(1,90)=6.52, p<.025]$, the generated-name condition $[F(1,90)=8.37, p<.005]$, and the veridicalname condition $[F(1,90)=10.46, p<.005]$ and was due to a number of false alarms in short-term memory being significantly higher for the no-learning condition than for the veridical-name condition $[F(1,90)=10.46, p<.005]$.
For discrimination scores, the ANOVA indicated significant variations of discrimination performance between the labeling condition $[F(4,90)=10.82, p<.0005]$ and the memory condition $[F(1,90)=11.35, p<.001]$. The interaction between these factors was not significant $[F(4,90)=1.88$, n.s. $]$. Orthogonal contrasts of means revealed discrimination scores significantly higher in shortterm memory for the veridical-name condition than for the other ones $(p<.0005)$ and in long-term memory for the no-learning, generated-name, and veridical-name conditions than for the no-label and chemical-name conditions $(.05>p<.0005)$. Thus, the apparent tendency in Figure 3 to observe progressive variations of hit and falsealarm scores (an increase in hits and a decrease in false alarms) from the no-label condition to the veridical-name condition (even when nonsignificant differences were noted between two adjacent means) was significantly confirmed when examining $d_{\mathrm{L}}^{\prime}$ scores-especially in the longterm condition. Discrimination scores were progressively better, going from the subjects who had no label to associate with odors to the subjects trained to associate chemical names with the odors to the subjects who generated names and, finally, to the subjects who used veridical names.

The analysis of bias measures indicated no significant variations as a function of the labeling factor $[F(1,90)=$ 1.68, n.s.] or as a function of the memory factor $[F(1,90)=$ 2.23 , n.s.], and there was no interaction between the nolabeling and memory conditions $[F(4,90)=0.48$, n.s. $]$. This was due to the fact that the interindividual variability for a same condition was too high to allow variations 
Correct recognition

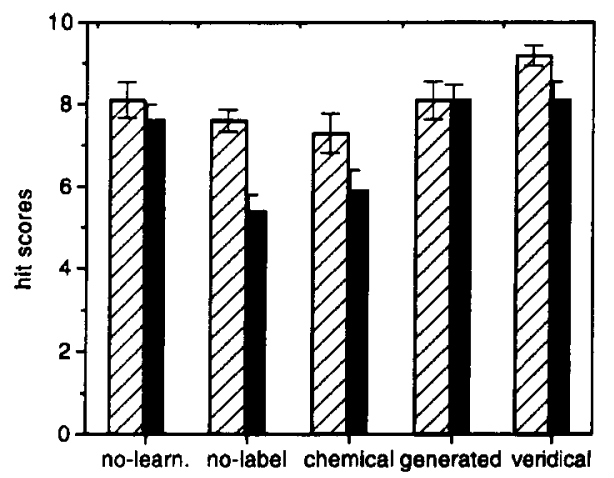

DD short term

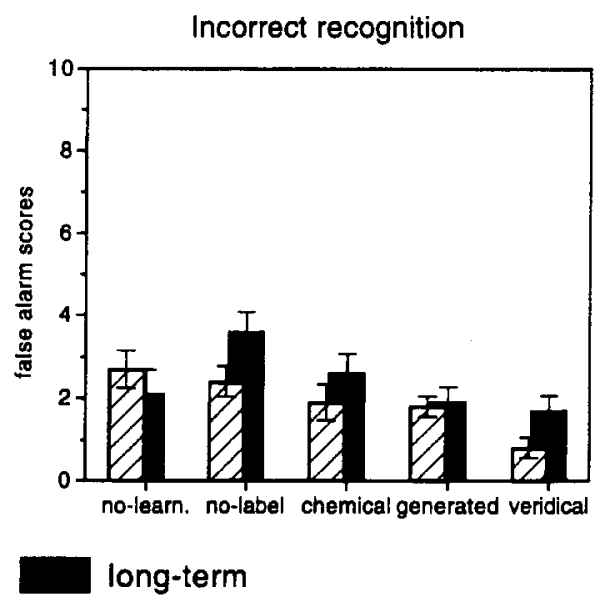

Bias measure

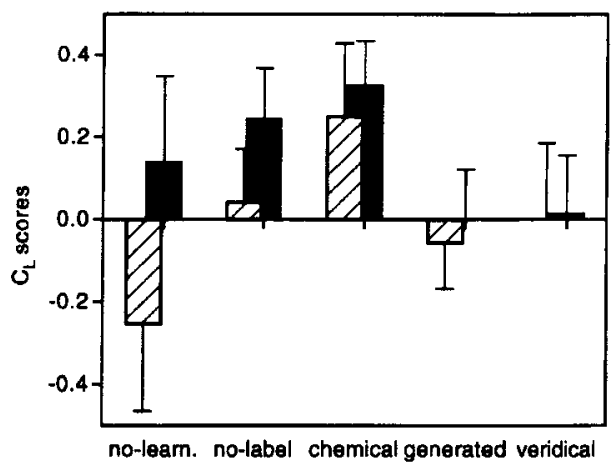

Figure 3. Representation of hit scores, false-alarm scores, discrimination scores $\left(d_{L}^{\prime}\right)$, and bias scores $\left(C_{\mathrm{L}}\right)$ in five experimental conditions: control condition without any learning (no-learn), sensory familiarization without any verbal learning (no-label), labeling with a chemical name (chemical), labeling with a personally generated name (generated), and labeling with a veridical name (veridical).

between different conditions to be observed (see $y$-axis, from -.255 to .325 ).

\section{Latency of Odor Recognition Memory Responses}

Latencies of odor recognition responses measured for target and distractor odors are represented as a function of different experimental factors in Figure 4 (top). A three-way ANOVA, with repeated measurements on the last factor, revealed neither significant effects of the memory condition (first factor) $[F(1,90)=2.76$, n.s. $]$ nor significant effects of the labeling condition (second factor) $[F(4,90)=$ 1.11, n.s.]; however, there was a significant difference of response latencies as a function of the nature of the tested odors (third factor, target vs. distractor) $[F(1,90)=8.00$, $p<.01]$. Orthogonal contrasts proved only a significant decrease of latency for the no-label condition in short-term memory. Although just one variation was significant, reliable results of the ANOVA could be explained by the recognition response latency being systematically shorter for distractor odors than for target odors, with the exception of the no-learning group in short-term memory.

These latencies for target and distractor odors were considered as a function of correctness of recognition responses - that is, correct (recognitions and rejections) and incorrect (misses and false alarms) responses (Figure 4, bottom). A three-way ANOVA, with repeated measurements on the last two factors (labeling condition and target vs. distractor), showed no significant variations due to memory conditions $[F(1,98)=.76$, n.s. $]$, a highly significant effect of the correctness of recognition response $[F(1,98)=46.15, p<.0005]$, and a small significant effect of the nature of the tested odors (target vs. distractor) $[F(1,98)=5.18, p<.025]$. Mean orthogonal contrasts proved that the latencies of recognition responses were significantly increased (on the average, from 5.61 to $9.48 \mathrm{sec}$ ) when the subjects made incorrect recognition responses (misses or false alarms) $(.001>p<.0005)$.

\section{Identification Performance}

The frequency of correct associations observed during each test $\left(\mathrm{IT}_{1}\right)$ of different blocks in the first learning session did not present significant variations along time, as revealed by a one-way ANOVA with repeated measurements $[F(3,27) \leq 2.72$, n.s.]. The four sets of data were therefore summed up and compared with frequencies of correct associations observed during the test of the second session $\left(\mathrm{IT}_{2}\right)$ and the test of the third session $\left(\mathrm{IT}_{3}\right)$. The results are depicted in Figure 5 as a function of the 

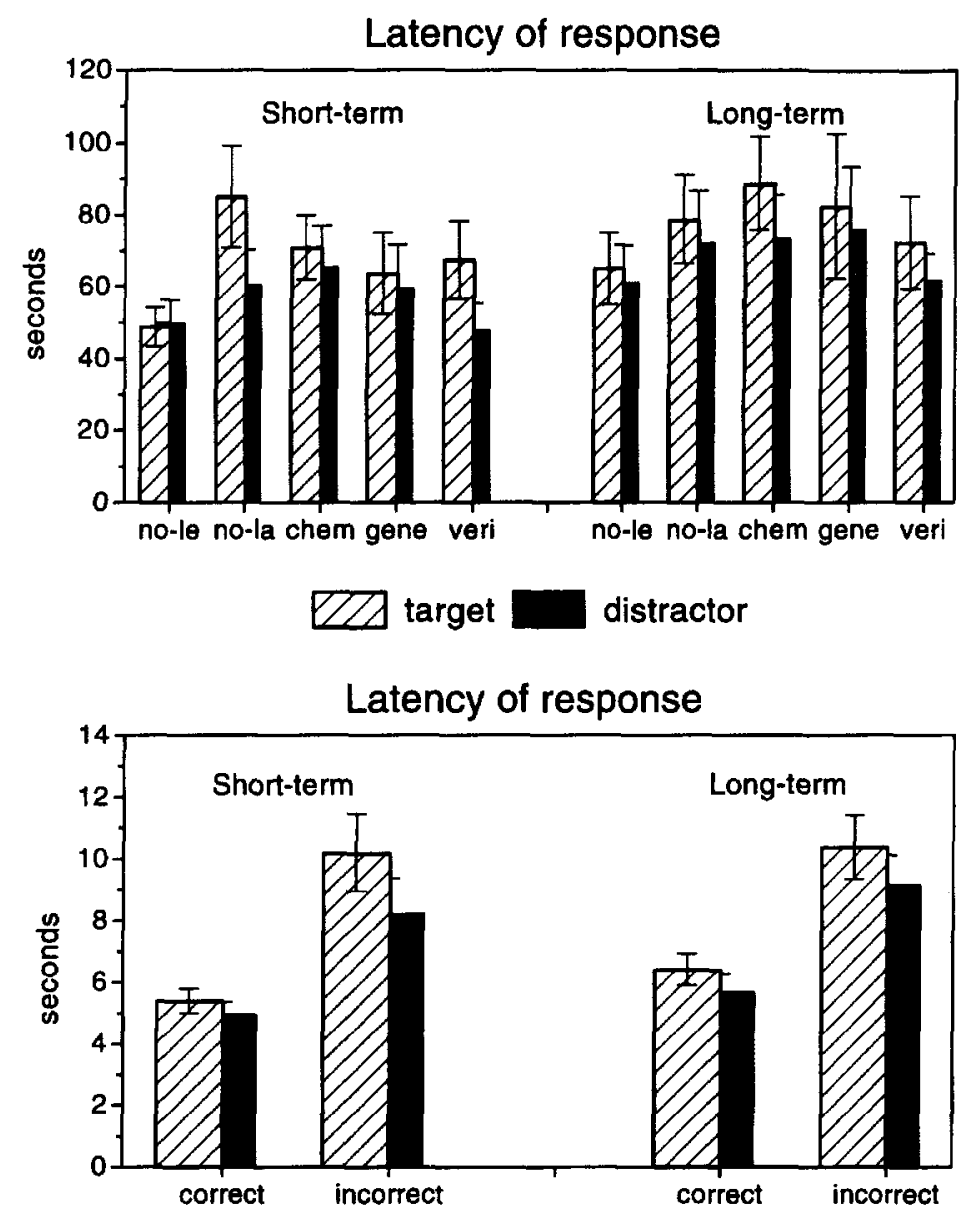

Figure 4. Top: Duration of response latencies in the odor recognition memory test as a function of the retention interval (short-term vs. long-term) and the five experimental conditions: no-learning (no-le), no-label (no-la), chemicalname (chem), generated-name (gene), and veridical-name (veri). Bottom: Duration of response latencies as a function of the retention interval (shortterm vs. long-term) and the accuracy of responses (correct or incorrect) in the memory test.

two memory conditions and the three labeling conditions. A three-way ANOVA, with repeated measurements on the third factor, revealed no reliable differences of correct identifications between short- and long-term memory tests (first factor) $[F(1,54)=1.40$, n.s. $]$, but significant variations of correct identifications as a function of labeling conditions (second factor) $[F(2,54)=33.54$, $p<.0005]$ and as a function of the three identification tests (first factor, $\mathrm{IT}_{1}, \mathrm{IT}_{2}$, and $\mathrm{IT}_{3}$ ) $[F(2,108)=171.28$, $p<.0005]$. Furthermore, multiple orthogonal comparisons of means, when grouped according to the labeling factor, showed, in the short-term condition, a significant increase of correct associations from chemical to veridical $[F(1,54)=15.77, p<.0005]$, from generated to veridical $[F(1,54)=8.31, p<.01]$, but no significant variation from chemical to generated $[F(1,54)=1.19$, n.s.]. In the long-term condition, these comparisons revealed a significant increase from chemical to generated $[F(1,54)=48.30, p<.0005]$ and from chemical to verid- ical $[F(1,54)=52.85, p<.0005]$. The more detailed examination of contrasts by pairs of means indicated a significant increase $(.05>p<.0005)$ of correct identifications from chemical to generated and from generated to veridical in most comparisons, in both memory conditions (except from generated to veridical for $\mathrm{IT}_{1}, \mathrm{IT}_{2}$, and $\mathrm{IT}_{3}$ in the long-term condition, and from chemical to generated or to veridical for $\mathrm{IT}_{1}$ in the short-term condition).

Finally, although some variations were not significant, the frequencies of correct identifications were notably and significantly reduced $(.05>p<.0005)$ from $\mathrm{IT}_{1}$ to $\mathrm{IT}_{2}$ and from $\mathrm{IT}_{2}$ to $\mathrm{IT}_{3}$, whatever the labeling condition considered (except between $\mathrm{IT}_{1}$ and $\mathrm{IT}_{2}$ for veridical labeling in the short- and long-term conditions).

\section{Verbal Memory Performance}

Verbal memory performances observed during the tests $V_{1}$ and $\mathrm{VT}_{2}$ (see Figure 1) are represented in Figure 6 as a function of memory and labeling conditions. 


\section{Correct identification}

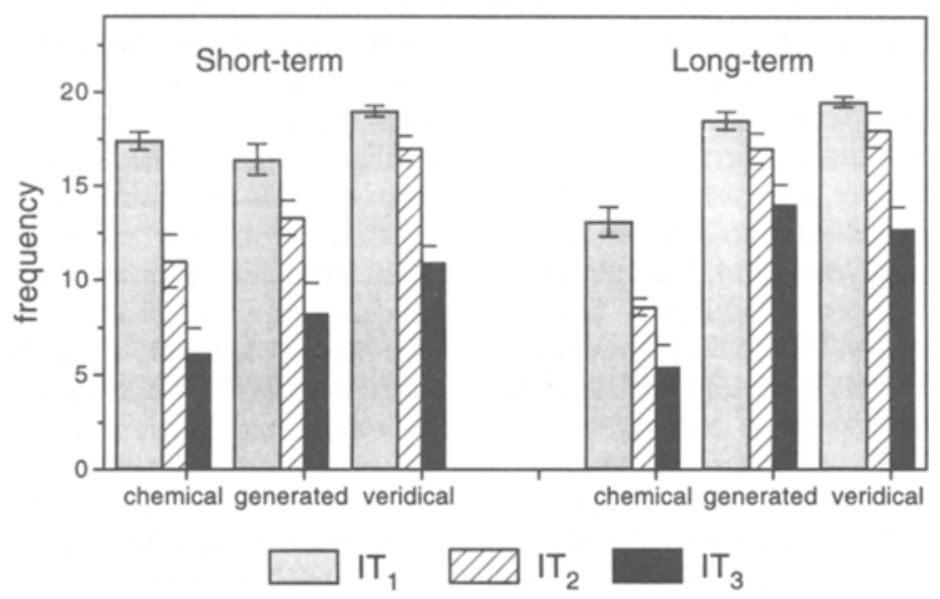

Figure 5. Frequency of correct responses for the three identification tests $\left(I T_{1}, I T_{2}\right.$, and $\left.I T_{3}\right)$ according to memory conditions (short-term and longterm) and the three labeling conditions (chemical-name, generated-name, and veridical-name).

The three-way ANOVA, with repeated measurements on the third factor, showed no significant variation between short- and long-term memory conditions $[F(1,54)=$ 0.00 , n.s.], a significant difference as a function of labeling conditions $[F(1,54)=11.14, p<.005]$, and a significant effect due to verbal memory test $\left(\mathrm{VT}_{1}\right.$ vs. $\left.\mathrm{VT}_{2}\right)$ $[F(1,54)=16.02, p<.0005]$. As evidenced by the analysis of contrasts, the verbal memory performance for the chemical-name condition was significantly reduced relative to that for the generated-name and veridical-name conditions for $\mathrm{VT}_{2}$ in the short-term memory and for $\mathrm{VT}_{1}$ and $\mathrm{VT}_{2}$ in long-term memory $[F(1,54)>15.6, p<.0005$, in all comparisons].
Furthermore, we noted a significant decrease of verbal memory performance from $\mathrm{VT}_{1}$ to $\mathrm{VT}_{2}$ for the chemicalname condition in short-term memory $[F(1,54)=30.62$, $p<.0005]$ and for the generated-name condition in longterm memory $[F(1,54)=4.90, p<.05]$.

\section{DISCUSSION}

\section{Odor Recognition Memory}

Influence of verbal encoding. The present study highlights the role of verbal encoding in olfactory recognition memory. In several conditions, the subjects who learned the names of odors while they were familiarized with them

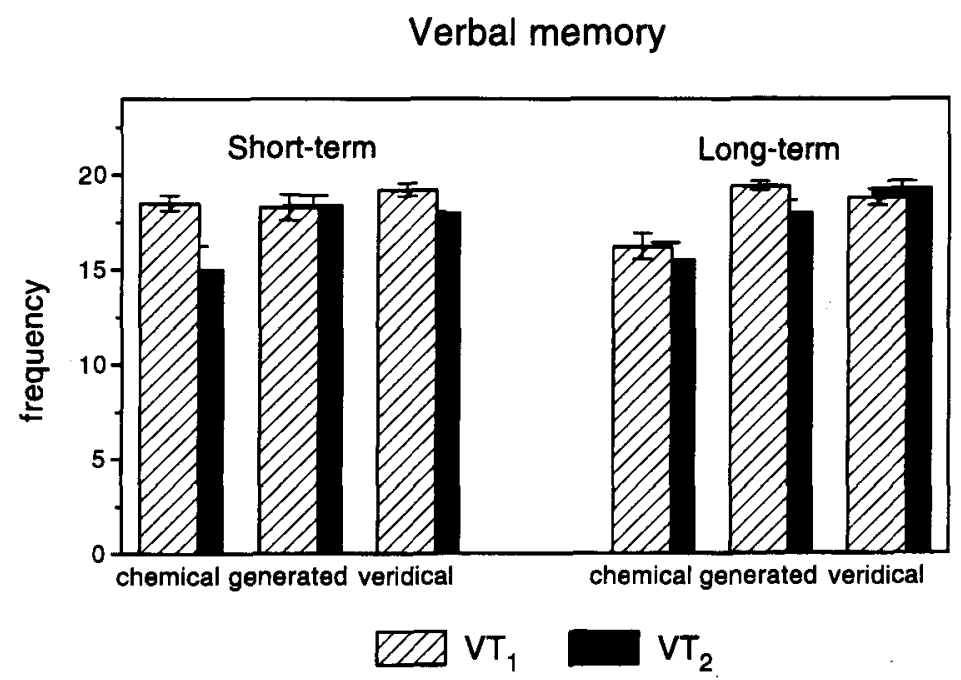

Figure 6. Frequency of correct recalls as a function of the two memory conditions (short-term and long-term) and the different categories of odor names (chemical, generated, and veridical) for verbal memory tests $V T_{1}$ and $V T_{2}$. 
exhibited higher discrimination between targets and distractors than did those who were only familiarized and those who were not familiarized at all. This resulted from an increase in the number of correct recognitions (hits) and a decrease in the number of false alarms.

These findings differ from those of an earlier study by Lawless and Cain (1975) but are in close agreement with observations from several other authors (Lyman \& McDaniel, 1986, 1990; Rabin \& Cain, 1984; Walk \& Johns, 1984). All of these investigators used highly familiar odors, such as those of common food substances or daily objects. Familiarity of these odors could be associated with the verbal labeling of some of them so that the two conditions - pure sensory familiarity and verbal codingwere potentially interacting. Because we investigated olfactory recognition using odors that were not familiar to subjects before training, our study was especially appropriate to dissociate familiarity and verbal encoding.

The main conclusion of this study - that is, the positive influence of verbal encoding on odor recognition-was not verified in several instances. In the short-term memory condition, the attribution of chemical names to odors did not improve olfactory memory performance, as compared with pure sensory familiarization. One may assume that chemical names were not mnemonically efficient for encoding target odors because, in contrast with veridical names, the link to odors they denote is of low semantic value. The results of odor identification $\left(\mathrm{IT}_{3}\right)$, which indicate lower performances for chemical names than for veridical and generated names, corroborate this interpretation. The olfactory recognition performance is therefore clearly related to the semantic content of the labels.

Response latencies. Response latencies have not often been used in olfaction (Köster \& De Wijk, 1991; Lawless \& Engen, 1977). The present study represents the first attempt to use response latencies to characterize olfactory recognition memory.

Recognition response latencies were systematically longer when the delivered odor was a target rather than a distractor. The reason could be that the delivery of the target odor calls for a positive response of recognition, whereas the delivery of the distractor calls for a negative response. It was hypothesized in a previous study (Jehl, Royet, \& Holley, 1994) that human beings are more inclined to look for differences in the odors to be compared than to seek their similarities. Declaring that two odors are different is possible as soon as one feature of the present perception is clearly distinct from the previously registered memories, whereas judging two odors as identical supposes a more expensive comparison in order to exclude all possibilities of dissimilarity. That response latencies were shorter when target and distractor odors were different is therefore understandable.

Moreover, when the recognition response was incorrect, the duration of the response latency was considerably longer $(9.48 \mathrm{sec})$ than when the response was correct $(5.61 \mathrm{sec})$. One may think that the subjects delayed their recognition judgment when they lacked certainty. The time of responses did not seem to vary as a function of the retention delay (short-term vs. long-term).

Influence of familiarization. It has been shown in a previous study (Jehl et al., 1995) that familiarization to odors resulted in an improved recognition memory for familiarized odors. The present study seems to lead to different conclusions given that the subjects who were familiarized with odors (no-labeling groups) displayed no enhanced recognition memory in comparison with the subjects in the control situation (no-learning group) and even produced lower scores (long-term odor memory). The reason for this discrepancy may be found in the large differences in the experimental protocols. They differed from each other in the length of the retention interval and the mode of odor delivery during the retention test. In the previous study, odors were presented in pairs with an interstimulus interval of $20 \mathrm{sec}$, whereas, in the present study, the odors were delivered in a set and the retention interval was $20 \mathrm{~min}$ or $24 \mathrm{~h}$. It may be assumed that the subjects erroneously interpreted test odors as "previously experienced," not because they had been presented with them in the first part of the test session but because they had been familiarized with them during the learning sessions. Presumably, the subjects were confused about the chronology of odor delivery. This confusion was still greater when the retention delay was $24 \mathrm{~h}$, which can explain the lower score of hits and the higher number of false alarms in this condition. The disturbing effects of recent familiarization could be counterbalanced only by the association of the most efficient label (i.e., the veridical name).

\section{Odor Identification and Verbal Memory}

The most remarkable observation regarding olfactory identification performances is the drastic drop of correct identifications from the first test $\left(\mathrm{IT}_{1}\right)$ to the third test $\left(\mathrm{IT}_{3}\right)$, regardless of the kind of name previously associated to the odors (Figure 5 and Table 2). This drop could express a decline of long-term stored olfactory and verbal information. This decrease as a function of time in spite of positive feedback during the first two sessions is surprising. Most authors have shown that subjects given feedback and training with the veridical names of odors that they could not previously identify showed a marked increased ability to identify these odors (Cain, 1979; Desor \& Beauchamp, 1974; Schemper, Voss, \& Cain, 1981). If the person identifies the object with its true name the first time it is presented, the probability of success will increase from $40 \%-50 \%$ to $80 \%-90 \%$ on a subsequent presentation. And, if the identification is only approximative, improvement will be only about $60 \%$ (Cain, 1982). Thus, we could at least suppose an increase in identification scores from $\mathrm{IT}_{1}$ to $\mathrm{IT}_{2}$ (i.e., from the first to the second day). In contrast, they decreased, on the average, by $20.4 \%$. It is possible that the identification score was adversely influenced by the complexity of our experimental procedure in which several tasks were performed during learning sessions, disturbing the effects of training. The higher reduction observed from $\mathrm{IT}_{2}$ to $\mathrm{IT}_{3}(-33.0 \%)$ 
Table 2

Percent Reduction of Performance in Different Labeling and Memory

Conditions Between the Three Identification Tests Compared in Pairs

\begin{tabular}{ccccccccc} 
& \multicolumn{3}{c}{ Short-Term Conditions } & & \multicolumn{3}{c}{ Long-Term Conditions } & \\
\cline { 2 - 4 } \cline { 6 - 7 } Ratio & Chemical & Generated & Veridical & & Chemical & Generated & Veridical & Mean \\
\hline $\mathrm{IT}_{2} / \mathrm{IT}_{1}$ & 36.8 & 18.9 & 10.5 & & 34.4 & 11.9 & 9.7 & 20.4 \\
$\mathrm{IT}_{3} / \mathrm{IT}_{2}$ & 44.5 & 38.3 & 35.9 & & 37.2 & 14.1 & 27.8 & 33.0 \\
$\mathrm{IT}_{3} / \mathrm{IT}_{1}$ & 64.9 & 50.0 & 42.6 & & 58.8 & 24.3 & 34.9 & 45.9 \\
\hline
\end{tabular}

can easily be explained as the decline of poorly consolidated associations during the delay of 6 days between Sessions 2 and 3.

One may assume that forgetting either olfactory or verbal information, or both, impeded identification of the delivered odor. However, verbal memory tests $\mathrm{VT}_{1}$ and $\mathrm{VT}_{2}$ did not reveal a significant forgetting (except a significant decrease for the chemical name in the short-term condition and for the generated name in the long-term condition). Our results of verbal memory indicate that during the third session $\left(\mathrm{VT}_{2}\right), 15-19.4$ names out of 20 previously presented were still remembered. The decrease of olfactory identification must, therefore, depend on the decline of odor memories themselves or the weakening of the links between names and odors.

The difference in verbal memory performance $\left(\mathrm{VT}_{1}\right)$ between short- and long-term groups for the chemicalname condition seems to have been due to an uncontrolled factor. The two groups of the subjects were submitted to the same experimental learning procedure. The different results could therefore be explained by intergroup variability due to the individuals themselves. Similarly, corroborating this interpretation, one can note that the correct identification score for the chemical-name condition $\left(\mathrm{IT}_{1}\right)$ was higher in short-term groups than in longterm groups.

\section{Perceptual Versus Semantic Encoding}

Comparisons of labeling and no-labeling discrimination performances, on the one hand, and odor identification and verbal memory performances, on the other hand, can allow the relative part of perceptual and semantic memories to be determined. In spite of training, and although demonstrating stable verbal memory, the subjects performed poorly in correct odor identification at the third session. On the average, only $9.55 \pm 3.54$ odor correct identifications in 20 were obtained in $\mathrm{IT}_{3}$, whereas
$17.32 \pm 2.35$ correct identifications in 20 were recorded in $\mathrm{IT}_{1}$. Mean reductions of identification performances ranged from $25.0 \%$ to $41.3 \%$ from the first to the third session according to the labeling groups. Nevertheless, verbal information seemed to minimize the loss of relevant information. If one compares discrimination (recognition) scores in long-term conditions versus short-term conditions (Table 3), one observes that the reduction of performances that reaches $63.6 \%$ in the absence of verbal labeling is only $44.0 \%$ with chemical names and no more than $31.4 \%$ with veridical labels. Thus, the semantic information seems to potentiate olfactory recognition performances.

This conclusion is in agreement with the general theory of dual coding proposed by Paivio (1986). The subjects who learned the odor names benefited from a double coding in that they became sensorially familiarized with odors, on the one hand, and they were provided with verbal labels denoting the odor character, on the other hand. Thus, one can assume that the odor memory trace was reinforced, thereby gaining durability.

According to Lyman and McDaniel (1990), odors being encoded twice rather than once (sensory) are far better retained over time. The explanation would be that a greater number of "retrieval pathways" give access to stored information. During the recognition test, the subjects benefit from two complementary ways to recognize a target odor. The first way is to compare the immediate olfactory perception with olfactory memories being stored during learning. The second way is to identify the delivered odor by a name and to check whether this name belongs to the list of those registered during learning.

Thus, one can assume that the level of odor retention depends on both stability and degree of semanticity of verbal marks used to denote odors. These characteristics are better represented by veridical and generated names than by chemical labels (as discussed earlier).

Table 3

Percent Reduction of Performance in Different Labeling Conditions Between the Two Recognition Tests (Long-Term/Short-Term), Two Identification Tests $\left(\mathrm{IT}_{3} / \mathrm{IT}_{1}\right)$, and the Two Verbal Memory Tests $\left(\mathbf{V T}_{\mathbf{2}} / \mathbf{V T}_{\mathbf{1}}\right)$

\begin{tabular}{|c|c|c|c|c|c|}
\hline \multirow[b]{2}{*}{ Test } & \multirow[b]{2}{*}{ Ratio } & \multicolumn{4}{|c|}{ Condition } \\
\hline & & No-Labeling & Chemical & Generated & Veridical \\
\hline $\operatorname{Recc}$ & long-term/short-term & 63.6 & 44.0 & 1.6 & 31.4 \\
\hline Identification & $\mathrm{IT}_{3} / \mathrm{IT}_{1}$ & n.m. & 41.3 & 25.0 & 31.8 \\
\hline Verbal memory & $\mathrm{VT}_{2} / \mathrm{VT}_{1}$ & n.m. & 12.1 & 3.45 & 1.84 \\
\hline
\end{tabular}

Note--n.m., not measured. 


\section{Conclusions}

The present study shows that olfactory recognition memory is enhanced through verbal encoding. This enhancement is apparent not only for the long-term retention period but also for the short-term retention period. The semantic content of the labels is clearly responsible for the mnemonic efficiency of verbal encoding. The verbal labels are all the more efficient because they are more liable to activate a background of representations that are congruent with the delivered stimuli, such as images of odor sources and autobiographic memories.

\section{REFERENCES}

BANKs, W. P. (1970). Signal detection theory and human memory. Psychological Bulletin, 47, 81-99.

CaIn, W. S. (1979, February 2). To know with the nose: Keys to odor identification. Science, 203, 467-470.

CAIN, W. S. (1980). Chemosensation and cognition. In H. van der Starre (Ed.), Olfaction and taste VII (pp. 347-357). London: IRL.

CAIN, W. S. (1982). Odor identification by males and females: Predictions vs. performance. Chemical Senses, 7, 129-142.

CoNOVER, W. J. (1971). Practical nonparametric statistics. New York: Wiley.

Craik, F. I. M., \& LockhaR'T, R. S. (1972). Levels of processing: A framework for memory research. Journal of Verbal Learning \& Verbal Behavior, 11, 671-684.

DAvis, R. G. (1977). Acquisition of verbal associations to olfactory stimuli of varying familiarity and to abstract visual stimuli. Journal of Experimental Psychology: Human Learning \& Memory, 3, 37-51.

Desor, J. A., \& BeAuchamp, G. K. (1974). The human capacity to transmit olfactory information. Perception \& Psychophysics, 16, 551-556.

DOTY, R. L., SNyder, P. J., Huggins, G. R., \& Lowry, L. D. (1981). Endocrine, cardiovascular, and psychological correlates of olfactory sensitivity changes during the human menstrual cycle. Journal of Comparative Physiological Psychology, 95, 45-60.

ENGEN, T. (1982). The perception of odors. New York: Academic Press.

EngEN, T. (1987). Remembering odors and their names. American Scientist, 75, 497-503.

Engen, T., Kuisma, J. E., \& Eimas, P. (1973). Short-term memory of odors. Journal of Experimental Psychology, 99, 222-225.

ENGEN, T., \& Ross, B. (1973). Long-term memory of odors with and without verbal descriptions. Journal of Experimental Psychology, 100, 221-227.

Jehl, C., Royet, J.-P., \& Holley, A. (1994). Very short term recognition memory for odors. Perception \& Psychophysics, 56, 658-668.

Jehl, C., RoYet, J.-P., \& Holley, A. (1995). Odor discrimination and recognition memory as a function of familiarization. Perception \& Psychophysics, 57, 1002-1011.
Köster, E. P., \& DE WIJK, R. A. (1991). Olfactory adaptation. In D. G. Laing, R. L. Doty, \& W. Breipohl (Eds.), The human sense of smell (pp. 199-215). Berlin: Springer-Verlag.

LAWLESS, H. T. (1978). Recognition of common odors, pictures, and simple shapes. Perception \& Psychophysics, 24, 493-495.

LAWLESS, H. T., \& CAIN, W. S. (1975). Recognition memory for odors. Chemical Senses \& Flavour, 1, 331-337.

LAWLESS, H. T., \& ENGEN, T. (1977). Associations to odors: Interference, mnemonics, and verbal labeling. Journal of Experimental Psychology: Human Learning \& Memory, 3, 52-59.

Lockhart, R. S., \& Murdock, B. B. (1970). Memory and the theory of signal detection. Psychological Bulletin, 74, 100-109.

Lyman, B. J., \& MCDANIEL, M. A. (1986). Effects of encoding strategy on long-term memory for odours. Quarterly Journal of Experimental Psychology, 38A, 753-765.

Lyman, B. J., \& MCDANiel, M. A. (1990). Memory for odors and odor names: Modalities of elaborating and imagery. Journal of Experimental Psychology: Learning, Memory, \& Cognition, 16, 656-664.

MuRPHy, C. (1985). Cognitive and chemosensory influences on agerelated changes in the ability to identify blended foods. Journal of Gerontology, 40, 47-52.

PaIvio, A. (1986). Mental representation: A dual coding approach. New York: Oxford University Press.

Rabin, M. D., \& CAIN, W. S. (1984). Odor recognition: Familiarity, identifiability, and encoding consistency. Journal of Experimental Psychology: Learning, Memory, \& Cognition, 10, 316-325.

Richardson, J. T. E., \& ZuCCO, G. M. (1989). Cognition and olfaction A review. Psychological Bulletin, 105, 352-360.

ScHAB, F. R. (1991). Odor memory: Taking stock. Psychological Bulletin, 109, 242-251.

SCHEMPER, T., Voss, S., \& CaIN, W. S. (1981). Odor identification in young and elderly persons: Sensory and cognitive limitations. Journal of Gerontology, 36, 446-452.

SCHNEIDER, R. A. (1974). New insights into the role and modifications of olfaction in man through clinical studies (Annals of the New York Academy of Sciences, Vol. 237, pp. 217-223). New York: New York Academy of Sciences.

ShEPARD, R. N. (1967). Recognition memory for words, sentences and pictures. Journal of Verbal Learning \& Verbal Behavior, 6, 156-163.

SNODGRASS, J. G., \& CoRwIN, J. (1988). Pragmatic of measuring recognition memory: Applications to dementia and amnesia. Journal of Experimental Psychology: General, 117, 34-50.

WALK, H. A., \& JoHNS, E. E. (1984). Interference and facilitation effects in short-term memory for odors. Perception \& Psychophvsics, 36, 508-514.

WINER, B. J. (1962). Statistical principles in experimental design. New York: McGraw-Hill.

(Manuscript received August 16, 1995; revision accepted for publication March 9, 1996.) 